\title{
The Maze Test: A Computer Tool for Testing Perseverance
}

This article was published in the following Dove Press journal:

Psychology Research and Behavior Management

\author{
Wojciech Styk (iD) \\ Waldemar Klinkosz (D) ${ }^{2}$ \\ 'Institute of Psychology, The John Paul II \\ Catholic University of Lublin, Lublin, \\ Poland; ${ }^{2}$ Institute of Psychology, Cardinal \\ Stefan Wyszynski University in Warsaw, \\ Warsaw, Poland
}

Introduction: Perseverance is an important component of human functioning. The main factors that allow people to achieve their goals are perseverance and the motivation for change. The concept of perseverance is not new, but most studies in this field do not define it precisely. Perseverance is a formal trait of behaviour determined by situational (external) and personalityrelated (internal) factors - a complexity which can cause problems when one tries to measure it. Methods: In this project, we present the Maze Test, a novel tool for testing perseverance. We discuss the theoretical foundations of the test, including the definition of perseverance, as well as the indicators on which this tool is based. We present the basic methodological properties of the test and report two studies which illustrate how it can be applied in practice: Study 1 - measurement of personality-conditioned perseverance, and Study 2 - measurement of situation-conditioned perseverance.

Results: In Study 1, a significant weak correlation between the indicators of perseverance and the Big Five model personality traits was discovered. Neuroticism correlated negatively with the number of maze tasks solved and the Synthetic Indicator of Perseverance. Positive correlations were found for conscientiousness and agreeableness. In Study 2, mental simulations were observed to have a medium to high effect on the indicators of perseverance.

Conclusion: The tool presented in this study affords a new approach to perseverance. Based on an analysis of how the tasks were being solved by the subjects, we developed perseverance indicators which allow to view perseverance as a multidimensional construct. The tool has been designed for use in both laboratory tests and remote web tests. A major advantage of the tool is that it is available for general use free of charge.

Keywords: perseverance, persistence, short-term goals, maze test

\section{Introduction}

"It's not that I'm so smart, it's just that I stay with problems longer."

-Albert Einstein

We deal with perseverance in human activity in situations in which people strive to achieve a goal despite disinclination to act or despite being distracted by external factors. Although there are many different theoretical approaches and terminological differences in the literature on this subject, research on perseverance in action is based on the assumption that setting a goal and accomplishing it are necessary but insufficient conditions to achieve goals. In empirical psychology, perseverance can be understood in three ways: as (1) a disposition, ie an individual property (related to personality traits) responsible for the continuity of action, which is independent of the situational context; (2) a behavioural feature, ie a trait of behaviour determined solely by external, situational factors; and (3)
Correspondence: Wojciech Styk Institute of Psychology, The John Paul I

Catholic University of Lublin, Al.

Raclawickie Street I4, Lublin 20-950,

Poland

Email wojciech.styk@gmail.com
Psychology Research and Behavior Management 2020:13 1277-1288 DovePress f in $\mathbf{P}$ 
a formal trait of behaviour determined by both situational (external) and personality-related (internal) factors. ${ }^{1}$

Although perseverance undoubtedly depends to a large extent on situational conditions, one can point to individual differences in perseverance. ${ }^{2}$ Some people achieve goals despite difficulties, while others fail. This assertion is confirmed by research which demonstrates that perseverance is a derivative of the power of will, because it reflects one's ability not to give up and fight on in spite of setbacks or failures. ${ }^{3}$

\section{Measurement of Perseverance Self-Report Measures of Perseverance}

Nowadays, perseverance is quite frequently used as a construct in psychological studies, but mainly as one of the scales of various types of inventories describing personality traits. An example of such an inventory is the Values in Action Inventory of Strengths (VIA-IS). In this measure, perseverance is defined as "finishing what one starts; persevering in a course of action in spite of obstacles; "getting it out the door"; taking pleasure in completing tasks". ${ }^{4}$ In a study by Littman-Ovadia and Shiri carried out in an international sample of 686 subjects, relationships between VIAIS strengths and performance at work were investigated. The results clearly demonstrated that there was a positive correlation (0.33) between perseverance and work efficiency, and a negative correlation $(-0.35)$ between perseverance and undertaking unproductive activities. The conclusion of that study was that in every profession, workplace, and organization, perseverance could be as important as talent and could lead to high productivity. ${ }^{5}$ However, perseverance is not just to do with work. Other studies have shown correlations of perseverance (also as a VIA-IS scale) with subjective wellbeing $(0.39)$, satisfaction with life (0.24), and positive affect (measured by PANAS) $(0.41){ }^{2}$

In recent years, many publications have been dedicated to the Grit Scale, which assesses trait-level perseverance, among other things. ${ }^{6-10}$ Grit is often described as a predictor of success and a construct which predicts performance better than other variables such as intelligence and/or conscientiousness. ${ }^{6,11,12}$ Grit consists of two independent factors. The first of them is consistency of interest, which can be defined as a tendency to adopt a particular long-term goal and the ability to concentrate one's activities around it. The second factor is perseverance of effort, which is understood as the capability to undertake long-term effort even in the face of adversity or failure. Consistency of interest is an indicator of the number of career changes. Perseverance of effort, on the other hand, predicts variables such as school grades, participation in non-compulsory activities, or the number of hours spent watching television. ${ }^{13}$ The Grit Scale can be used to predict success in areas commonly considered as difficult or requiring hard work. ${ }^{14}$ High scorers on the Grit Scale are able to persistently pursue long-term objectives even if they encounter difficulties and obstacles and their efforts do not bring the expected results at the outset. ${ }^{12}$ A study by Maddi et al has shown that perseverance of effort and consistency of interest are associated with courage as they require individuals to effectively cope with stressful situations and hence actively deal with adversities. ${ }^{15}$

The relationship between perseverance and selfconsciousness has been studied by Auzoult and collaborators. The questionnaire they used contained one item related to perseverance: "I try to achieve what I decide whatever the difficulty". They found a positive correlation $(0.18)$ between perseverance and self-consciousness. ${ }^{16}$

\section{Measuring Perseverance Through Practical Tasks}

Perseverance is also known to experimental psychology, but the methods of its measurement are not consistent.

In an experiment in which the subjects solved word anagrams until they independently decided to give up on the task, researchers found that providing solutions, or just ensuring that a solution will be delivered at the end of the task reduced the subjects' perseverance. The authors of that study used task solving time (when the participants new the answer was available) as the indicator of perseverance. ${ }^{17}$

In another study, investigating the relationship between the testosterone level and perseverance, the subjects had to solve puzzles. Initially they received a solvable task and then an unsolvable puzzle. In that experiment, the investigators adopted the time of solving the unsolvable task as the indicator of perseverance. ${ }^{18}$

The relationship of stress and controlled and uncontrolled failures with perseverance was studied using a computer game which simulated earning an academic degree. Perseverance, defined as continuity of action in achieving a goal despite failure, was calculated as the percent of choices for the same path right after facing a defeat on this path. The investigators drew a distinction between setbacks that were dependent on the subject ("failed exam") and those that were independent of the subject ("course cancellation"). ${ }^{19}$ Perseverance research also explores perseverance in achieving long-term goals measured in months or years. An example of this is the study on Afro-American students and their perseverance in 
continuing education, where the indicator of perseverance was the time of continuing education. ${ }^{20}$

Interesting research on effectiveness and perseverance was also conducted by Marszał-Wiśniewska and Jarczewska-Gerc, who investigated the importance of mental simulations in the process of losing weight. In one of their experiments they measured the subjects' perseverance as the number of logins to the website with the survey. The study participants had been asked to log on to the survey website as often as possible (eg every day) and record their current weight. ${ }^{21}$

In a study by Ilmari Määttänen et al, the authors assessed, in a laboratory setting, the presence of evolutionarily relevant behavioural tendencies as well as the participants' ability and motivation to continue an unpleasant behaviour. They investigated two separate perseverance factors: "physical" and "mental". The physical factor was strongly correlated with a hand grip endurance task and a cold presser task. The mental factor was strongly associated with a verbal reasoning task. A thread and needle task and a boring video task were both correlated, rather strongly, with both factors, however the correlation was slightly stronger for the mental factor. A task with an unsolvable anagram was weakly, but significantly, associated with the mental factor. ${ }^{22}$

The above overview of research shows that there is no unambiguous definition of perseverance in the literature. The different approaches to and definitions of perseverance may indeed indicate that it is a multidimensional construct. The indicators of perseverance used in the literature include: (a) perseverance in solving simple and repetitive tasks, where there is no negative stimulus associated with failure; (b) perseverance in solving tasks that require tenacity, despite a negative stimulus associated with failure, and (c) perseverance in achieving short-term and long-term goals. Worth noting is also the distinction drawn between persistence and perseverance in research on perseverance as a motivational factor. This research includes both self-description studies and the latest experimental studies.

In this paper, we present the Maze Test, a new tool for measuring perseverance that has been made available for use to the general public free of charge. We report preliminary results of validity and stability studies and describe the indicators of perseverance that this tool measures. Additionally, we describe two experiments that illustrate the usefulness of the test and exemplify its possible applications. In these experiments perseverance is treated as either a permanent trait or a situationally determined characteristic The examples were chosen to show how broad a concept perseverance is. We hope that the Maze Test will gain popularity and that the results of studies carried out using this test will provide additional data, such as norms and scales, and help advance the research on perseverance.

\section{The Maze Test Definition of Perseverance}

As indicated in the Introduction, studies on perseverance use different definitions of the concept or do not provide a precise definition at all. For the sake of accuracy, however, it is necessary to precisely define the concept so that it is clear what type of perseverance is involved (eg, perseverance in pursuing long-term vs short-term goals). The focus of this present study is perseverance in pursuing short-term goals in the absence of a negative stimulus related to task difficulty. When designing the tool, we assumed that the indicators of perseverance will be measured using simple recurrent labyrinth tasks. The method of measuring perseverance by determining its various indicators in the performance of repetitive tasks has been applied in several studies. ${ }^{6,18,23}$ In these publications, perseverance was defined as the ability to overcome discomfort resulting from the desire to give up, overcoming emerging obstacles such as distractors and uncontrolled distractive thoughts, and the ability to control the desire to engage in substitute activities. In our study, we also adopted this definition of perseverance.

\section{Design of the Computer Tool}

The Maze Test includes a collection of over 150 mazes. It was designed to include easy tasks so that the subjects should not have negative feelings about failing to solve the tasks. As defined by Łukaszewski: easy tasks are those in which $80 \%$ of the subjects achieve at least $80 \%$ correctness of result (...), difficult tasks are those in which $80 \%$ of people attain correctness not higher than $25 \%{ }^{23}$

In order to facilitate the use of the tool, a dedicated website was created. The site was built to work with modern web browsers. Practical tests have shown that the Maze Test site works with Firefox, IE/Edge, Chrome, and Safari browsers.

To do the test, the user needs to have a computer mouse. The Maze Test does not work properly on touch screens. The website uses "cookies", which is why each test should be done in a separate window in the private 
mode. After completing the test and closing the window saved during the test, "cookies" are removed and the next subject can start the test with the browser's blank memory.

To start the test, the user needs to open the browser window in the private mode, and enter the website address for the test. The subject is then asked to move the mouse cursor to indicate the path from the green square to the red square, bypassing obstacles ("black walls"). An example of a maze is shown in Figure 1.

When the correct route is indicated on the screen, another maze is displayed. The sequential mazes are displayed until the subject gives up and clicks the "abort the game" button. The mazes are displayed in a random order. They are selected with replacement, which means that there is no upper limit to the number of mazes to be solved, and the same maze can be displayed more than once.

Before proceeding to the task proper, the subject has the opportunity to solve a sample labyrinth. In our pilot study conducted on 131 people, $98 \%$ of the respondents solved no more than 200 mazes.

The current version of the tool is available free of charge at www.mazetest.org for non-commercial use.

Figure 2 shows a screenshot of the start page, in which users can set the test parameters, such as test time or number of mazes. Figure 3 presents a screenshot of a maze task being solved.

\section{Indicators of Perseverance}

The Maze Test allows to determine perseverance in action using the following indicators:

Number of maze tasks solved - an indicator which defines perseverance in completing simple and repetitive tasks taking into account the efficacy of solving them.

Time spent on solving the tasks - an indicator which defines perseverance in completing simple and repetitive tasks without considering efficacy but only the time spent on the activity.

Synthetic Indicator of Perseverance (SIP) - an auxiliary indicator calculated as the quotient of the number of tasks solved and the average solving time for one task. This indicator combines perseverance understood in terms of efficacy of solving tasks and resistance to distractors, as well as the capacity to inhibit irrelevant thoughts and/or memories. High SIP values are obtained when the respondents are strongly focused on the task and solve a lot of mazes. Low SIP values are observed when the participants are not sufficiently focused on the task and/or are

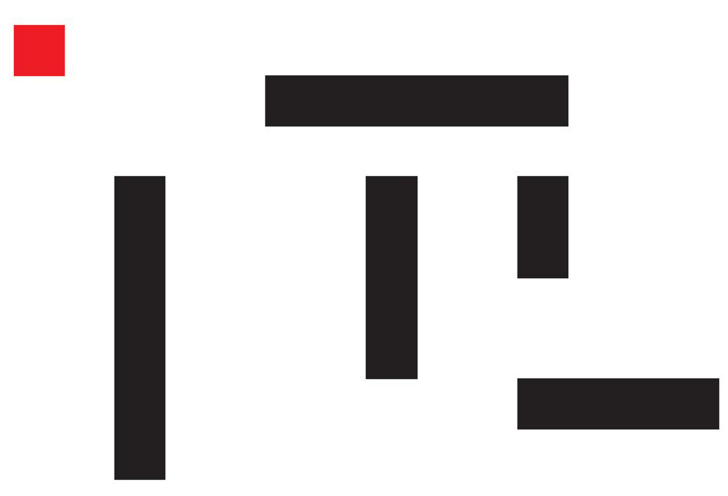

Figure I An example of a maze task.

subjected to distractors (activities or thoughts) and hence solve a low number of tasks.

In addition, the Maze Test measures the average time spent on one task, which is an auxiliary index related to focusing. It is calculated as the quotient of the time spent on a task and the number of tasks solved. The focusing index determines both the ability to ignore distractors and the ability to suppress irrelevant thoughts or memories. It reaches high values when the individuals are not focused enough on the task at hand and are exposed to activities or thoughts that distract them from the task being performed. Conversely, low values of the focusing index show that the subject has managed to avoid being distracted from the task. Low scorers have the ability to suppress irrelevant thoughts and distractions.

\section{Validity of the Maze Test Participants and Procedure}

Participants were recruited through a dedicated website. The address of the website was provided to students of The John Paul II Catholic University of Lublin, Poland. No eligibility restrictions were placed on the participants. The only condition for enrolling in the study was provision of consent to participate. A lack of consent made it impossible for a person to go to the next screens containing the Maze Test.

The website provided information about the study and its goal and contained a short description of the study procedure. The subjects were informed that by clicking "Next" they agreed to participating in the study. At the outset, each participant completed a demographic survey inquiring about gender, age, and level of education. In the next step, the subjects read instructions for completing the tasks. The test consisted in solving a series of simple mazes. The 


\section{mazetest.org}

\section{Test}

Results

Items and scales

Publications

A step by step - How to use

Licence to use

About us

Contact

\section{The Maze Test}

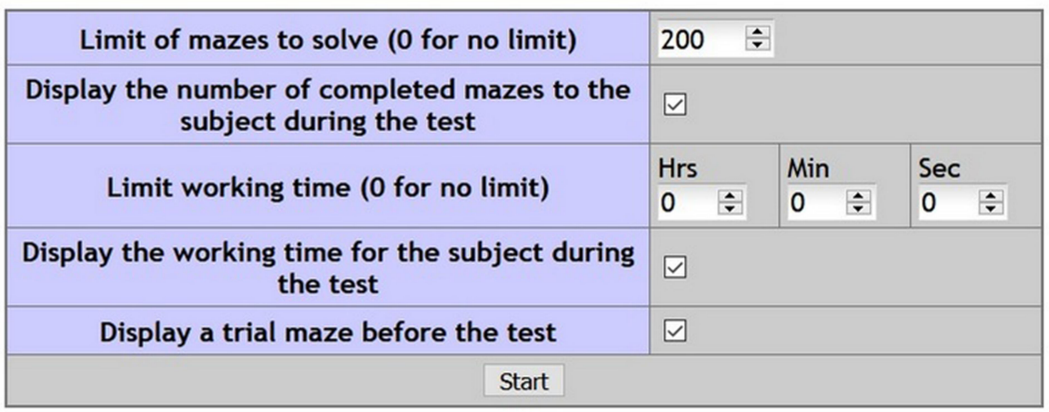

Figure 2 Start page of the Maze Test.

\section{mazetest.org}



STOP

Figure 3 Screenshot of a maze task being solved.

subjects were asked to solve as many maze tasks as possible without any time limit. A maze was solved by marking with a mouse cursor (pointer) the path from a green point ("entrance") to a red point ("exit"), bypassing black "walls". The participants had the opportunity to solve a trial maze before they proceeded to the task proper. The maze 
tasks were displayed one by one (one maze per screen). Once the participant found his way through the maze, they were presented with another, randomly allocated maze. They could end a task at any time by clicking the "Quit" button. At the end of each task, a screen saying "Thank you for participating in the study" was displayed.

The study involved 135 participants (including 64\% women, $\mathrm{n}=87$ ), mean age of 32 years (range 19-50 years). Most of the subjects had a higher education degree $(82 \%)$.

In the next step, the participants were recruited as above, except that in addition to completing the tasks on The Maze Test (described above), they were asked to complete another task. The instruction for that task contained information that the goal of the task was to collect preliminary data for the next task in which problemsolving skills were tested. The task was adapted from Welker and Carre as quoted by Baumeister et al (1998). ${ }^{18}$ The puzzle required the participants to draw a geometric shape without tracing over any lines or lifting the pen. The participants were given two solvable puzzles to solve; all the remaining puzzles were unsolvable. The subjects were instructed to solve as many puzzles as they wanted and to click the "Quit" button if they no longer wished to continue the task.

The test was completed by 48 subjects ( $54 \%$ women, $\mathrm{n}=26$ ), mean age of 28 years.

\section{Convergent Validity}

In the first step, The Perseverance (PE) Scale from the Achievement Motivation Inventory (AMI) self-report questionnaire was used to assess the convergent validity of The Maze Test. ${ }^{24}$ The PE scale of AMI consists of 10 items. The authors of AMI define perseverance as endurance, and the use of strengths to complete the tasks set by themselves or by others. People who achieve high scores on this scale have the ability to focus on their tasks, and maintain a sufficient level of energy for tasks. They are able to direct their full attention to the task at hand and are not easily discouraged from completing an important task (op. cit.). Pearson's correlation coefficient ( $r$ ) between the scores on the PE scale of the self-report questionnaire and PE indicators in The Maze Test was $0.23(\mathrm{p}<0.001)$ for the number of tasks solved and $0.256(\mathrm{p}<0.001)$ for the task solving time spent.

In the second step, the convergent validity of The Maze Test was established using a practical unsolvable puzzle test, in which the amount of time spent on the unsolvable puzzles served as a measure of perseverance. ${ }^{18}$ Pearson's correlations between time spent on solving the unsolvable puzzles and the other variables used in the analysis were as follows: 0.87 (p $<0.01)$ for time spent solving The Maze Test, $0.77(p=0.04)$ for number of Maze tasks solved, and $0.55(\mathrm{p}<0.05)$ for SIP.

\section{Stability}

The reliability of measurement was calculated using the test-retest method. Two weeks after the first administration, 30 participants were asked to perform the Maze Test again. On the basis of these data, correlations between perseverance coefficients obtained two weeks apart were calculated. The short-term stability index in the Maze Test was $0.55(\mathrm{p}<0.01)$ for the number of tasks solved, and $0.45(\mathrm{p}<0.01)$ for the time spent on completing a task.

As the literature indicates, the self-control construct is diversely operationalized in psychological research. According to Duckworth and Kern's meta-analysis of the convergent validity of behavioral and self-report measures of self-control, these tools demonstrate moderate convergence $\left(r_{\text {random }}=0.27[95 \% \mathrm{CI}=0.24,0.30] ; \mathrm{r}_{\text {fixed }}=0.34\right.$ $[0.33,0.35], \mathrm{k}=282$ samples, $\mathrm{N}=33,564$ participants). The authors of that study found that self-control is a coherent but multidimensional construct best assessed using multiple methods. They concluded that there was sufficient evidence for the convergent validity of selfcontrol measures, and that it was as strong as the evidence for the convergent validity of other psychological measures. ${ }^{25,26}$ Although the levels of correlation between the Maze Test scores and the PE scale are not high, they do not differ from those obtained in Duckworth and Kern's meta-analysis. Therefore, they should be considered as sufficient and confirming the convergence of the method.

The analysis of these results shows that the Maze Test is an valid and reliable test that has a satisfactory diagnostic value.

\section{Application Study I - Personality-Conditioned Perseverance}

In this experiment, we examined the relationship between personality, as represented by the five factor model, and perseverance in the action. In psychology, factor-analysis has been used since the 1970s to identify the basic variables that appear in the description of personality, ie personality traits, or personality dimensions. The existence of these traits can be deduced by observing the behaviour and emotional responses of human beings. ${ }^{27}$ Despite many 
uncertainties regarding the number of traits, in practice, researchers most commonly use five-factor model which include factors such as neuroticism, extraversion, openness to experience, agreeableness, and conscientiousness. These traits are understood as coherent patterns of individual differences in thinking, feeling, and behaving. ${ }^{28}$ A study conducted in a group of 184 people showed that perseverance, measured as a factor in the International Personality Item Pool - Values in Action (IPIP-VIA) questionnaire, correlated with the Big Five model factors. ${ }^{2}$ In this present experiment, we wanted to examine the relationships between personality traits and the indicators of perseverance measured by the Maze Test.

\section{Participants and Procedure}

The test was completed by 346 people, $56 \%$ of females $(\mathrm{N}=141)$ and $44 \%$ of males $(\mathrm{N}=111)$. The youngest subject was 18 years old, and the oldest was 58 years old. The mean age was 35 years. The study was conducted using a dedicated website. Participants were recruited through on-line forums, e-mail invitations, and social networking sites. No limits were placed on the number of subjects. Only those subjects who gave their consent to participating in the study were enrolled and could proceed to the actual test. The website also contains a note about the aim of the study and information on the test procedure as described in Participants and Procedure.

\section{Measures}

Personality traits of the subjects were determined using Costa and McCrae's NEO-FFI personality inventory. NEO-FFI, which is based on the five-factor model of personality, is frequently used in employee training and vocational counselling as well as in professional development and personality development. ${ }^{29}$ An abbreviated version of Costa and McCrae's inventory contains 60 items (12 items per scale) and allows to make a general diagnosis of the five basic personality factors. The subjects' perseverance was measured using the Maze Test.

\section{Results and Discussion}

The correlations of the perseverance indicators with the Big Five personality dimensions (Neuroticism, Extroversion, Openness to experience, Conscientiousness, and Agreeableness; measured by NEO-FFI) are shown in Table 1. Neuroticism was significantly negatively correlated with the number of maze tasks solved and SIP. Agreeableness and Conscientiousness were significantly correlated only with the number of maze tasks solved. The correlations ranged from 0.119 to to 0.163 . The other correlations turned out to be statistically non-significant. Peterson and Seligman have found that there are some clear theoretical links between persistence and self-regulation, which reflect qualities included in Conscientiousness. ${ }^{30}$ In our study the only indicator that did not take into account the efficacy of solving tasks was task solving time, which was correlated with Conscientiousness. Indicators which represent the efficacy of solving tasks (number of maze tasks solved and SIP) were negatively correlated with Neuroticism. Littman-Ovadia also found a negative correlation of persistence with Neuroticism $(-0.12)$, and a positive one with Agreeableness (0.19). ${ }^{2}$ These results provide a basis for further research on perseverance as a multidimensional concept.

\section{Study 2 - Situation-Conditioned Perseverance}

In this experiment, we investigated the impact of mental simulations on perseverance in action. Mental simulations are a special form of mental imagery, defined as imitative representations of an event or a series of events. ${ }^{31}$ They can be divided into three basic types: (1) outcome simulations, ie, representations of the final effect of an action; (2) process simulations, ie representations of an action plan and actions leading to the achievement of an objective; and (3) meditations, ie negative images of failure and anxiety. ${ }^{32}$ Process simulations may facilitate effective goal-orientation, while outcome simulations and meditations interfere with selfregulation. 1,23,33,34 The results of research by Marszal-

Table I Correlations Between Perseverance Indicators and Big Five Personality Dimensions

\begin{tabular}{|l|l|l|l|l|l|}
\hline & N & E & O & A & C \\
\hline Number of maze tasks solved & $-0.142^{* *}$ & 0.005 & 0.014 & $0.180^{* *}$ & 0.093 \\
Time spent on solving the tasks & -0.030 & 0.058 & 0.036 & 0.095 & $0.163^{* *}$ \\
Synthetic Indicator of Perseverance & $-0.119^{*}$ & -0.023 & -0.001 & 0.073 & 0.001 \\
Average time spent on one task & 0.057 & 0.053 & -0.019 & 0.057 & 0.002 \\
\hline
\end{tabular}

Notes: ${ }^{* *} \mathrm{p}<0.01 ;{ }^{*} \mathrm{p}<0.05$.

Abbreviations: $\mathrm{N}$, neuroticism; $\mathrm{E}$, extroversion; $\mathrm{O}$, openness to experience; $\mathrm{C}$, conscientiousness; $\mathrm{A}$, agreeableness. 
Wisniewska and Jarczewska-Gerc on the role of mental simulations in weight loss processes clearly show that imagining the process of body mass loss increases both perseverance and the effectiveness of the intended action. ${ }^{21,35}$ In our study, we used outcome and process simulations. We predicted that these simulations would affect the perseverance indicators measured by The Simple Maze Test.

\section{Participants and Procedure}

The test was completed by 252 people, 141 of females $(56 \%)$ and 111 of males (44\%). The youngest subject was 18 years old, and the oldest was 58 years old. The mean age was 35 years. The study was conducted using the dedicated website. Participants were recruited through on-line forums, e-mail invitations, and social networking sites. No restrictions were placed on the number of participants. Only those individuals who gave their consent to participating in the study were enrolled and could proceed to the actual test. The website also contains a note about the aim of the study and a description of the test procedure. The study was divided into two stages. In the first stage the subjects completed a survey on sex, age and education level. In the second stage, the subjects were randomly assigned either to the outcome-oriented mental simulation group, or the processoriented mental simulation group. The subjects were presented with a task to be solved and received instructions. The task consisted in solving a series of simple mazes, and the subjects were required to solve as many maze tasks as possible without any time limit. Before the task was started, the participants were subjected to one of the two simulations, having first received instructions relevant for a given group. The instructions for the outcome simulation focused the subjects' attention on the outcome of their work and contained the following sentences: "imagine that you get the best result in this task", "focus on the result", "think only about the result", "imagine that you have eventually achieved your goal - you are the best", "your friends will congratulate you on the result and will be impressed you have achieved such a high score". The instructions for process simulation focused the subjects' attention on the task solving process and contained the following sentences: "imagine that you walk through the maze", "try to imagine how you solve it", "imagine every move one by one", "think whether there is a better solution".

\section{Measures}

The subjects' perseverance was measured using the Maze Test.

\section{Results and Discussion}

The experiment showed that mental simulations had a statistically significant effect of on the indicators of perseverance. The effect of mental simulations on the average time spent on one task (focusing index) was not significant. The results are shown in Table 2 and Figure 4. The respondents from the process simulation group solved almost 50\% more labyrinth tasks than the respondents from the outcome simulation group. Process simulation was also found to influence the indicators that did not take into account the efficacy of solving the tasks (ie the time spent on solving the tasks). The respondents from the process simulation group dedicated more time to solving the tasks than the respondents from the outcome simulation group. For these comparisons, a medium effect size was observed, with Cohen's $d$ in the range of $0.37-0.43 .^{36}$ Mental simulations had the largest effect size on SIP. The subjects from the process simulations group achieved higher SIP values/scores compared to the subjects from the outcome simulations group. A vast effect size was observed. ${ }^{36}$ This study confirmed the influence of mental simulations on perseverance in action. The results are

Table 2 Perseverance and Mental Simulations. Group Characteristics and Results of Student's t-Test

\begin{tabular}{|c|c|c|c|c|c|c|c|c|}
\hline Perseverance Indicators & Mental Simulations & $\mathbf{N}$ & $\mathbf{M}$ & SD & $\mathbf{t}$ & df & $\mathbf{p}$ & Cohen's d \\
\hline Number of maze tasks solved & $\begin{array}{l}\text { Result } \\
\text { Process }\end{array}$ & $\begin{array}{l}128 \\
124\end{array}$ & $\begin{array}{l}63.17 \\
90.10\end{array}$ & $\begin{array}{l}53.27 \\
89.47\end{array}$ & -2.91 & 250.00 & $<0.01$ & 0.365 \\
\hline Time spent on solving the tasks & $\begin{array}{l}\text { Result } \\
\text { Process }\end{array}$ & $\begin{array}{l}128 \\
124\end{array}$ & $\begin{array}{l}473.89 \\
687.43\end{array}$ & $\begin{array}{l}361.46 \\
611.18\end{array}$ & -3.39 & 250.00 & $<0.01$ & 0.425 \\
\hline Synthetic Indicator of Perseverance & $\begin{array}{l}\text { Result } \\
\text { Process }\end{array}$ & $\begin{array}{l}128 \\
124\end{array}$ & $\begin{array}{l}9.59 \\
12.70\end{array}$ & $\begin{array}{l}10.20 \\
14.64\end{array}$ & -1.96 & 250.00 & 0.05 & 6.591 \\
\hline Average time spent on one task & $\begin{array}{l}\text { Result } \\
\text { Process }\end{array}$ & $\begin{array}{l}128 \\
124\end{array}$ & $\begin{array}{l}9.62 \\
9.30\end{array}$ & $\begin{array}{l}4.63 \\
10.20\end{array}$ & 0.42 & 210.98 & 0.67 & - \\
\hline
\end{tabular}



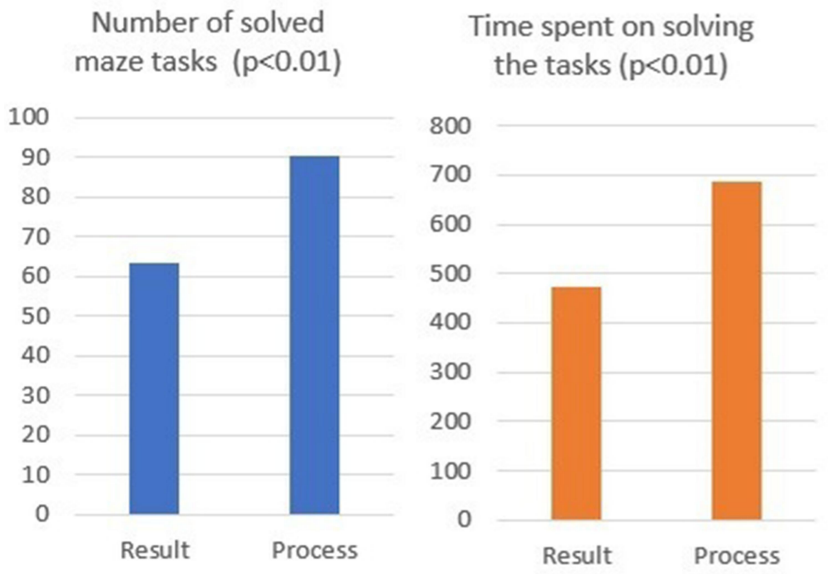

Figure 4 Perseverance and mental simulations.

consistent with previous studies using other methods of perseverance measurement. ${ }^{1,23,33,34}$

These results provide a basis for further research on the influence of mental simulations on perseverance. The Maze Test could be used in studies on other mental simulations, eg various simulations. The impact of these simulations has been studied using such perseverance indicators as the number of logins or weight loss when slimming. ${ }^{21}$ However, the effect of these simulations on persistence as a multidimensional index has not yet been investigated.

\section{General Discussion}

Perseverance helps people achieve their goals throughout the lifetime, starting from childhood, when the first walking attempts result in falls, through college years, when students have to learn complex science concepts until late at night, to old age, when we need it, for example at work. Because the tasks we have to do are often boring and the gratification is postponed, success requires perseverance and the capability to combat frustration and the desire to abandon the activity. ${ }^{37}$ The ability to cope with these obstacles has been linked to intelligence, school achievements, and professional success. ${ }^{38}$ Therefore, it is important to understand how perseverance develops and what factors affect it.

The present article reports preliminary findings regarding the methodological properties and the applicability of the Maze Test. Our results confirm that perseverance should be viewed in two ways: as a trait determined by personality and as a situation-dependent characteristic. The experiments we conducted show that the Maze Test is universal in this respect and works for both approaches.

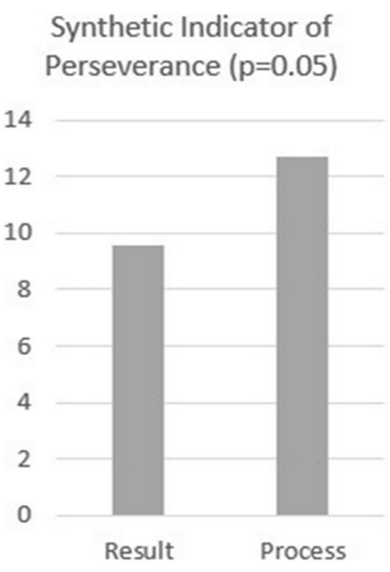

Average time spent

on one task $(p=0.67)$

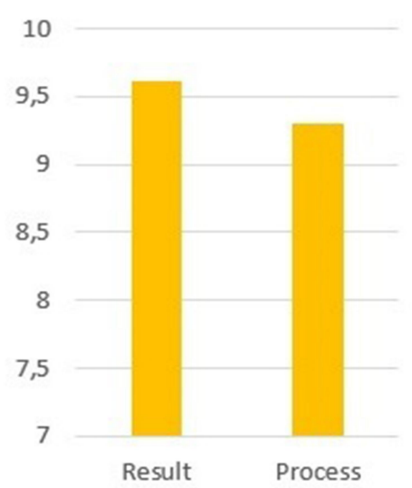

The Maze Test affords a new structured approach to perseverance testing. Based on the analysis of the way the participants solved the mazes, we developed perseverance indicators which make it possible to study it as a multidimensional construct. The perseverance measures used so far do not have uniform, standardized indicators, which makes it difficult to compare the results. Some studies use indicators based solely on the task solving time while others use indicators that take into account the efficiency of solving a task. ${ }^{18,22,35,39}$ The Maze Test uses operationalized persistence indicators which allow the researcher to consider, or ignore, the efficiency of solving a task. A Synthetic Indicator of Perseverance and a unique focusing index were also developed.

The results of the two tests we conducted confirm that the Maze Test can be used in both experimental and correlational research. In Study 1, a significant weak correlation was found between the indicators of perseverance and the Big Five personality traits. Neuroticism correlated negatively with the number of maze tasks solved and SIP. These indicators correlated positively with conscientiousness and agreeableness. Littman-Ovadia et al reported similar correlation values for perseverance measured using a self-descriptive questionnaire. ${ }^{2}$ This demonstrates that our tool has good validity. Study 2 demonstrated that the Maze Test can be used to measure perseverance as a situationally-conditioned characteristic. Mental simulations were found to exert an effect on the indicators of perseverance. The effect size ranged from medium to high. The research on mental simulations conducted so far has focused mainly on perseverance in achieving long-term goals. ${ }^{23,35}$ Our research shows that mental simulations influence perseverance in achieving short-term goals. 
The Maze Test has the great advantage of not requiring specialized equipment. It has been designed for use with commonly available computer hardware and software. Owing to this, researchers will be able to use the same operationalized tool and indicators to obtain repeatable and comparable measurements.

Elliot, Maier, Moller, Friedman and Meinhardt (2007) have shown that a two-second perception of red before a task can activate avoidance motivation and impair performance. ${ }^{40}$ Steele and Aronson (1995) have demonstrated that the mere act of reporting ethnicity prior to a test may activate a self-fulfilling negative stereotype that influences the test-taker's performance. ${ }^{41}$ These studies confirm that the knowledge of perseverance and the subtle factors that affect it is incomplete and deserves further investigation. The Maze Test can be used in this line of research, as described in our previous study on the relationship between body image and perseverance, in which the first version of the test was used. ${ }^{39}$ The results of that study showed that perseverance is associated with body image. There was a correlation between the distractor resistance index and BMI. Lower values of the perseverance indexes were observed in people who perceived their body weight as too high but not necessarily overweight.

To recapitulate on the results obtained in this present study, it should be emphasized that they highlight important aspects of both perseverance and efficacy. A vital role is played here by the interaction of situational and personality factors, which means that only diversified studies taking into account individual dispositions of the subjects can expand our knowledge in this area. The Maze Test is a tool that fits in perfectly with current research on perseverance. Providing free and open access to this simple and operationalized tool should facilitate research and at the same time improve its quality, allowing researchers to use the same multidimensional indicators and a single tool across a wide range of studies.

\section{Limitations and Conclusions}

A limitation of the Maze Test is that it requires access to the Internet and the use of a computer mouse with a pointer. When using the tool, one should remember about external factors that may affect the subjects' ability to solve the tasks and/or distract the subjects. These factors should be eliminated to meet the experimental conditions. When conducting experiments using The Maze Test, one should also pay attention to the distinction between persistence and perseverance, which in some special cases may constitute separate factors. ${ }^{42}$ As it stands, the tool does not allow to differentiate between these two constructs, and so separate tests should be used to discriminate between these factors wherever necessary.

The Maze Test can be used to determine perseverance in both experimental and correlation studies. The fact that the subjects can be given various types of instructions before they start solving the mazes offers a wide spectrum of possibilities of application. A great advantage of this tool is that it has operationalized perseverance indicators (which take into account, or ignore, efficacy), a synthetic persistence indicator and a unique focusing index. The tool is available for non-commercial use at www.mazetest.org.

\section{Ethical Issues}

The study was approved by the Ethics Committee of the Catholic University of Lublin, according with the ethical standards established by the Helsinki Declaration. The study participants provided written informed consent.

\section{Acknowledgments}

Special thanks are due to Georgia Lucas and Christiana Lucas for proofreading and editing assistance, as well as verification of the content of the article.

\section{Disclosure}

The authors declare that the research was conducted in the absence of any commercial or financial relationships that could be interpreted as a potential conflict of interest.

\section{References}

1. Marszał-Wiśniewska M, Jarczewska-Gerc E. Self-Control in Weight Loss Process. In: Ignacio Jauregui Lobera (ed.)Weight Loss. IntechOpen Limited; 2018:13-40. doi:10.5772/intechopen.76127

2. Littman-Ovadia H, Lavy S. Character strengths in Israel: hebrew adaptation of the VIA inventory of strengths. Eur J Psychol Assess. 2012;28(1):41-50. doi:10.1027/1015-5759/a000089

3. Park N, Peterson C, Seligman M. Strengths of character and well being. J Soc Clin Psychol. 2004;23(5):603-619. doi:10.1521/ jscp.23.5.628.50749

4. Zucker SH, Corley KM. Character strengths and intellectual and developmental disability: a strengths-based approach from positive psychology. Educ Train Autism Dev Disabil. 2012;47(2):13-25. doi:10.1073/pnas.0703993104

5. Littman-Ovadia H, Lavy S. Going the extra mile: perseverance as a key character strength at work. $J$ Career Assess. 2015;1-13. doi:10.1177/1069072715580322

6. Datu JAD, Valdez JPM, King RB. Perseverance counts but consistency does not! Validating the short grit scale in a collectivist setting. Curr Psychol. 2016;35(1):121-130. doi:10.1007/s12144-015-9374-2

7. Rimfeld K, Kovas Y, Dale PS, Plomin R. True grit and genetics: predicting academic achievement from personality. J Pers Soc Psychol. 2016;111(5):780-789. doi:10.1037/pspp0000089 
8. Credé M, Tynan MC, Harms PD. Much ado about grit: a meta-analytic synthesis of the grit literature. $J$ Personal Soc Psychol. 2017;113(3):492-511. doi:10.1037/pspp0000102

9. Wyszyńska P, Ponikiewska K, Karaś D, Najderska M, Rogoza R. Psychometric properties of the polish version of the short grit scale. Polish Psychol Bull. 2017. doi:10.1515/ppb-2017-0026

10. Disabato DJ, Goodman FR, Kashdan TB. Is grit relevant to well-being and strengths? Evidence across the globe for separating perseverance of effort and consistency of interests. J Pers. 2018. doi:10.1111/jopy.12382

11. Datu JAD, Yuen M, Chen G. Grit and determination: a review of literature with implications for theory and research. J Psychol Couns Sch. 2016;27:02. doi:10.1017/jgc.2016.2

12. Duckworth AL, Peterson C, Matthews MD, Kelly DR. Grit: perseverance and passion for long-term goals. J Pers Soc Psychol. 2007;92 (6):1087-1101. doi:10.1037/0022-3514.92.6.1087

13. Duckworth AL, Quinn PD. Development and validation of the short Grit Scale (Grit-S). J Pers Assess. 2009;91(2):166-174. doi:10.1080/ 00223890802634290

14. Von Culin KR, Tsukayama E, Duckworth AL. Unpacking grit: motivational correlates of perseverance and passion for long-term goals. $J$ Posit Psychol. 2014;9(4):306-312. doi:10.1080/ 17439760.2014.898320

15. Maddi SR, Erwin LM, Carmody CL, Villarreal BJ, White M, Gundersen KK. Relationship of hardiness, grit, and emotional intelligence to internet addiction, excessive consumer spending, and gambling. J Posit Psychol. 2013;8(2):128-134. doi:10.1080/ 17439760.2012 .758306

16. Auzoult L, Kubiszewski V, Hardy-Massard S. Perseverance in the Effort: A new standard of self-regulation associated to private self-consciousness. Swiss J Psychol. 2016;75(4):182-187. doi:10.1024/1421-0185/a000184

17. Risko EF, Huh M, McLean D, Ferguson AM. On the prospect of knowing: providing solutions can reduce persistence. J Exp Psychol Gen. 2017;146(12):1677-1693. doi:10.1037/xge0000334

18. Welker KM, Carré JM. Individual Differences in Testosterone Predict Persistence in Men. Eur J Pers. 2015;29(1):83-89. doi:10.1002/ per. 1958

19. Bhanji JP, Kim ES, Delgado MR. Perceived control alters the effect of acute stress on persistence. J Exp Psychol Gen. 2016;145 (3):356-365. doi:10.1037/xge0000137

20. Proctor SL, Nasir A, Wilson T, Li K, Castrillon P. Retention and persistence of African-American students in school psychology programs. Psychol Sch. 2018;55(5):506-522. doi:10.1002/pits.22124

21. Marszał-Wiśniewska M, Jarczewska-Gerc E. Role of mental simulations in the weight loss process. J Psychol Interdiscip Appl. 2016;150 (1):1-14. doi:10.1080/00223980.2014.987102

22. Määttänen I, Makkonen E, Jokela $M$, et al. Evidence for a behaviourally measurable perseverance trait. bioRxiv. 2020 doi:10.1101/2020.05.05.079509

23. Lukaszewski W, Jarczewska-Gerc E. Mental Simulation and Persistence in Action. J Russ East Eur Psychol. 2012;50(3):26-46. doi:10.2753/RPO1061-0405500302

24. Schuler H, Thornton III GC, Frintrup A, Mueller-Hanson R. AMI: Achievement Motivation Inventory. Technical and User's Manual. Hogrefe \& Huber Publishers; 2004.

25. Duckworth AL, Kern ML. A meta-analysis of the convergent validity of self-control measures. $J$ Res Pers. 2011;45(3):259-268. doi:10.1016/j.jrp.2011.02.004
26. Eisenberg IW, Bissett PG, Zeynep Enkavi A, et al. Uncovering the structure of self-regulation through data-driven ontology discovery. Nat Commun. 2019;10(1):1. doi:10.1038/s41467-019-10301-1

27. McCrae RR, John OP. An Introduction to the Five-Factor Model and Its Applications. J Pers. 1992;60(2):175-215. doi:10.1111/j.14676494.1992.tb00970.x

28. Whiteside SP, Lynam DR. The five factor model and impulsivity: using a structural model of personality to understand impulsivity. Pers Individ Dif. 2001;30(4):669-689. doi:10.1016/S0191-8869(00) 00064-7

29. Klinkosz W, Iskra J. Examination of the relations of the myers-briggs type indicator and the neo-4 personality inventory in a polish sample. Psychol Rep. 2010;107(2):578-586. doi:10.2466/08.09. PR0.107.5.578-586

30. Peterson C, Seligman M Character Strengths and Virtues: A Handbook and Classification; 2004. Available from: http://psychia tryonline.org/doi/abs/10.1176/appi.ajp.162.4.820-a.

31. Taylor SE, Schneider SK. Coping and the Simulation of Events. Soc Cogn. 1989;7(2):174-194. doi:10.1521/soco.1989.7.2.174

32. Simulation M, Taylor SE, Pham LB, Rivkin ID, Armor DA. Harnessing the imagination. Mental simulation, self-regulation, and coping. Am Psychol. 1998;53(4):429-439. doi:10.1037/0003066X.53.4.429

33. Sheeran P, Harris P, Vaughan J, Oettingen G, Gollwitzer PM. Gone exercising: mental contrasting promotes physical activity among overweight, middle-aged, low-SES fishermen. Heal Psychol. 2013;32(7):802-809. doi:10.1037/a0029293

34. Oettingen G, Schwörer B. Mind wandering via mental contrasting as a tool for behavior change. Front Psychol. 2013;4:(SEP):1-5. doi:10.3389/fpsyg.2013.00562

35. Marszał-Wiśniewska M. Self-control in weight loss process. In: Lobera EJ-GE-IJ, editor. IntechOpen. 2018. doi:10.5772/ intechopen.76127

36. Sawilowsky SS. New Effect Size Rules of Thumb. J Mod Appl Stat Methods. 2009;8(2):597-599. doi:10.22237/jmasm/1257035100

37. Duckworth AL, Kirby TA, Tsukayama E, Berstein H, Ericsson KA. Deliberate Practice Spells Success. Soc Psychol Personal Sci. 2011;2 (2):174-181. doi:10.1177/1948550610385872

38. Meier G, Albrecht MH. The Persistence Process: development of a Stage Model For Goal-Directed Behavior. J Leadersh Organ Stud. 2007;10(2):43-54. doi:10.1177/107179190301000205

39. Styk W, Zmorzyński S, Klinkosz W. Is body image associated with perseverance? A study among people with normal and increased body weight. Psychol Res Behav Manag. 2019;12:851-860. doi: $10.2147 /$ prbm.s215440

40. Elliot AJ, Maier MA, Moller AC, Friedman R, Meinhardt J. Color and psychological functioning: the effect of red on performance attainment. J Exp Psychol Gen. 2007;136(1):154-168. doi:10.1037/ 0096-3445.136.1.154

41. Steele CM, Aronson J. Stereotype threat and the intellectual test performance of African Americans. J Pers Soc Psychol. 1995;69:5. doi:10.1037/0022-3514.69.5.797

42. Serpell L, Waller G, Fearon P, Meyer C The Roles of Persistence and Perseveration in Psychopathology.; 2008. www.elsevier.com/locate/ bt. 


\section{Publish your work in this journal}

Psychology Research and Behavior Management is an international, peer-reviewed, open access journal focusing on the science of psychology and its application in behavior management to develop improved outcomes in the clinical, educational, sports and business arenas. Specific topics covered in the journal include: Neuroscience, memory and decision making; Behavior modification and management; Clinical applications; Business and sports performance management; Social and developmental studies; Animal studies. The manuscript management system is completely online and includes a very quick and fair peer-review system, which is all easy to use. Visit http://www. dovepress.com/testimonials.php to read real quotes from published authors. 